\title{
Monolithic Metal-Coated Plastic Components for mm-Wave Applications
}

\author{
A. von Bieren*, E. de Rijk*, J.-Ph. Ansermet ${ }^{\dagger}$, A. Macor*, \\ *SWISSto12 SA, EPFL Innovation Park, 1015 Lausanne, Switzerland \\ ${ }^{\dagger}$ Institute of Condensed Matter Physics, EPFL, 1015 Lausanne, Switzerland
}

\begin{abstract}
A stereolithography-based manufacturing process for monolithic high aspect ratio components for mm-wave and sub-mm-wave applications is demonstrated. A $25 \mathrm{~mm}$ long straight waveguide and a diagonal horn antenna, both for the WR-3.4 band (220-330 GHz), are manufactured and characterized. The waveguide is found to exhibit transmission losses close to the theoretical minimum for $\mathrm{Cu}$, and the performance of the diagonal antenna in terms of cross-polarization and directivity matches closely a metallic split-block reference antenna. These results confirm the high surface quality and mechanical accuracy of the employed 3D printing and plating techniques and thus validate the process for rapid manufacturing of monolithic components up to $330 \mathrm{GHz}$.
\end{abstract}

\section{INTRODUCTION}

A DDITIVE manufacturing has recently been introduced as a viable alternative to conventional manufacturing techniques for passive microwave (MW) and millimeter-wave (MMW) components, offering short lead times, drastically reduced weight and enhanced design flexibility [1]. In particular methods that are based on stereolithography (SLA) are considered a strong contender as these currently provide among the highest resolution and the best surface properties of suitable additive manufacturing techniques.

SLA parts are made of a non-conductive photopolymer. Therefore, inner surfaces and waveguide flanges have to be metallized subsequent to the printing process. MMW rectangular waveguides, feed horns, filters and other hollow passive components exhibit high aspect ratio channels that can be challenging to metallize. Previous studies relied on the splitblock technique where the component is split into two or more pieces that are separately coated and subsequently aligned and assembled [2]. This is an expensive and time-consuming process that often leads to degraded performance.

Here we present a manufacturing process that provides unprecedented performance for monolithic high aspect ratio components and hence avoids the aforementioned issues. To demonstrate its suitability for MMW components, a straight waveguide and a diagonal antenna for the WR-3.4 band are compared with their conventionally machined counterparts.

\section{FABRICATION}

The employed fabrication process is based on highresolution SLA with a nominal layer thickness of $25 \mu \mathrm{m}$. Microscope measurements of the as-printed parts show a lateral geometrical accuracy of lower than $\pm 10 \mu \mathrm{m}$. After appropriate mechanical and chemical pre-treatment steps, the parts are selectively metallized using a custom-developed copper plating process. Special emphasis has been placed on optimizing the plating processes for the photopolymer used. As a last step,

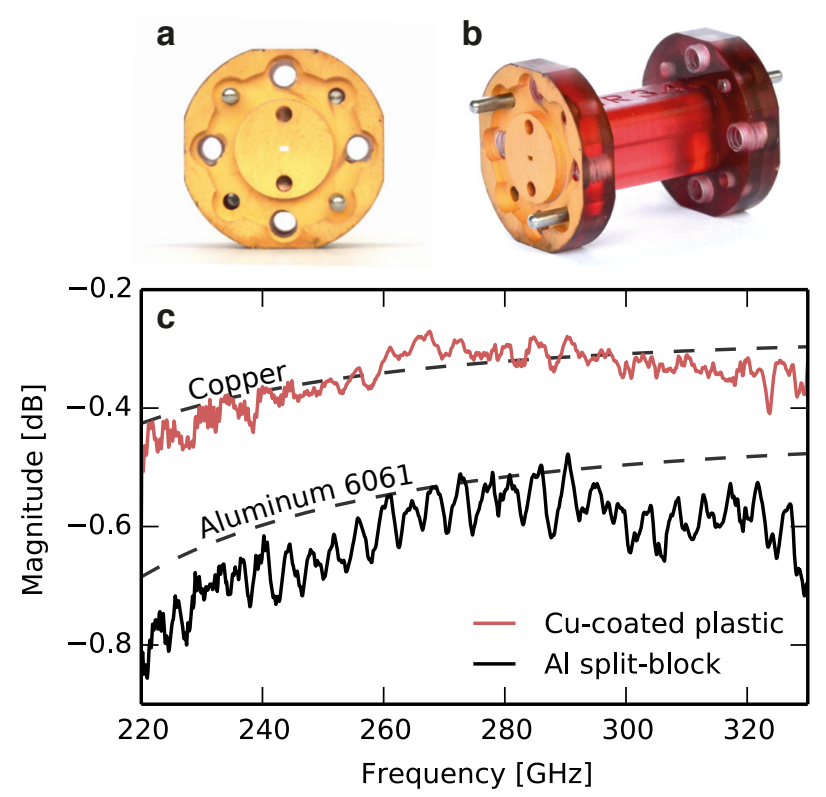

Fig. 1. $25 \mathrm{~mm}$ long rectangular waveguide prototype for the WR-3.4 band made of metal-coated plastics. (a) Front view of the metal-coated UG-387/UM flange, and (b) 3D view of the complete waveguide. Note that the metal-coated waveguide channel can be recognized and hence inspected from the outside due to the translucence of the used resin. (c) Measured transmission $\left(\mathrm{S}_{21}\right)$ of a $25 \mathrm{~mm}$ long plastic waveguide compared to a 1-inch aluminum alloy reference waveguide. Dashed lines show the theoretical attenuation of a WR3.4 rectangular waveguide for $\mathrm{Al} 6061$ and copper.

the waveguide flanges are passivated using a thin $(\sim 100 \mathrm{~nm})$ layer of gold.

\section{RESULTS}

All measurements shown are performed using an Agilent N5224A Vector Network Analyzer equipped with frequency extender modules from VDI for 220 to $330 \mathrm{GHz}$. Antenna radiation pattern measurements are carried out by mounting one of the extender modules on a 4-axis translation stage. Using a short pick-up waveguide, phase and relative amplitude of the electric field are sampled at arbitrary points in space.

\section{A. Straight Waveguide}

As a first benchmark component, a $25 \mathrm{~mm}$ long fundamental rectangular waveguide for the WR-3.4 band (220 to $330 \mathrm{GHz}$, $864 \times 432 \mu \mathrm{m}^{2}$ waveguide aperture) has been manufactured [see Fig. 1(a-b)]. The component is compared with a $25.4 \mathrm{~mm}$ long split-block aluminum alloy waveguide. As shown in Fig. 1(c), the signal attenuation in the $\mathrm{Cu}$-coated plastic waveguide is roughly $50 \%$ lower than in the reference waveguide. This 

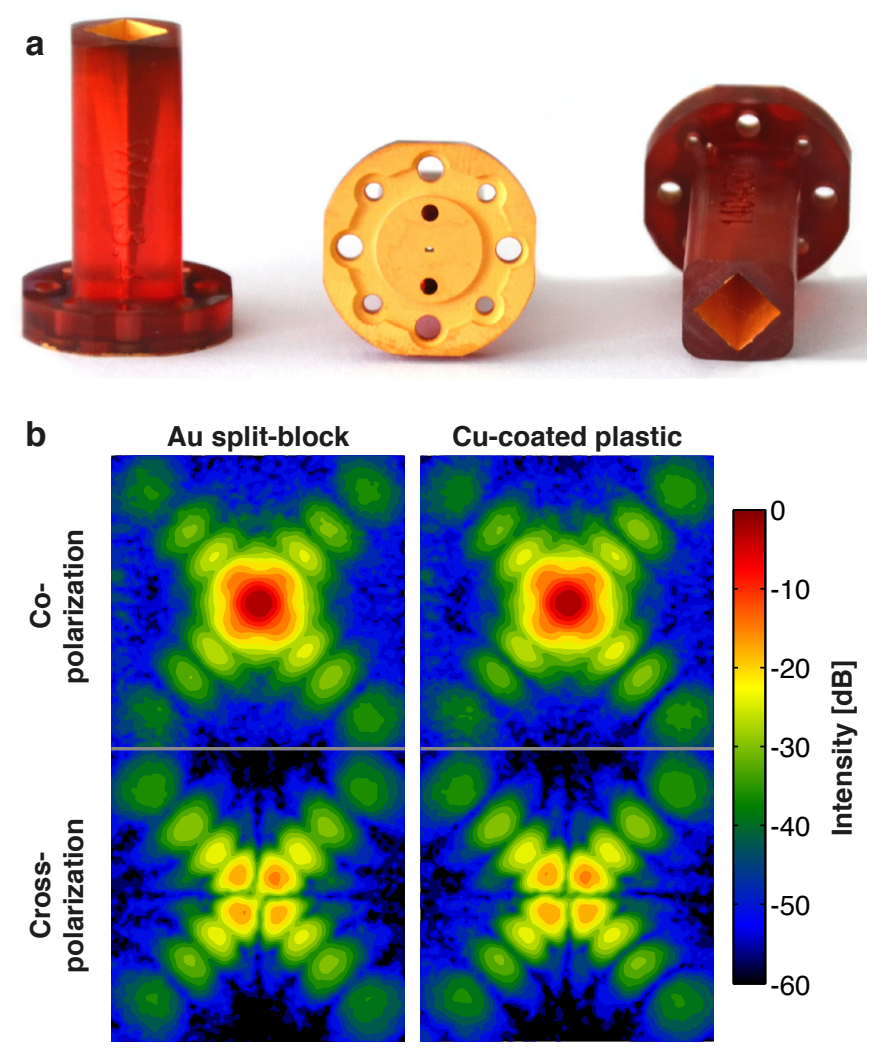

Fig. 2. (a) Pictures of the $\mathrm{Cu}$-coated plastic diagonal horn (from left to right: side-view, WR-3.4 flange, output aperture) before mounting the alignment pins. (b) Comparison of co- and cross-polarization radiation patterns at $330 \mathrm{GHz}$ of the reference horn (left column) and the $\mathrm{Cu}$-coated plastic horn (right column). Each picture shows a $200 \times 200 \mathrm{~mm}^{2}$ beam cross section measured at a distance of $100 \mathrm{~mm}$ from the output aperture.

can mainly be attributed to the difference in electrical conductivity between the aluminum alloy (Al 6061) of the splitblock waveguide and the plated copper. Importantly, similar to the metallic waveguide, the metal-coated plastic waveguide performs very close to its theoretical limit [see dashed lines in Fig. 1(c)]. This highlights the achieved mechanical accuracy, surface quality and coating homogeneity.

\section{B. Diagonal Horn Antenna}

Additionally, a WR-3.4 smooth-walled diagonal horn antenna [3] has been manufactured [see Fig. 2(a)]. In the following, its radiation characteristics is compared with a machined Au-coated split-block horn that is based on the same design.

Fig. 2(b) shows a selection of beam cross sections at the upper band edge $(330 \mathrm{GHz})$ where the mechanical precision is most crucial. The cross sections were taken at a distance of $100 \mathrm{~mm}$ from the output aperture for both the co-polarization and the cross-polarization content of the beam. The agreement between the metallic reference horn and the $\mathrm{Cu}$-coated plastic horn is striking: Side lobes down to $-40 \mathrm{~dB}$ are accurately reproduced by the plastic horn in terms of angular position, size and intensity. The measured cross-polarization at the center of the beam is even slightly better for the plastic horn

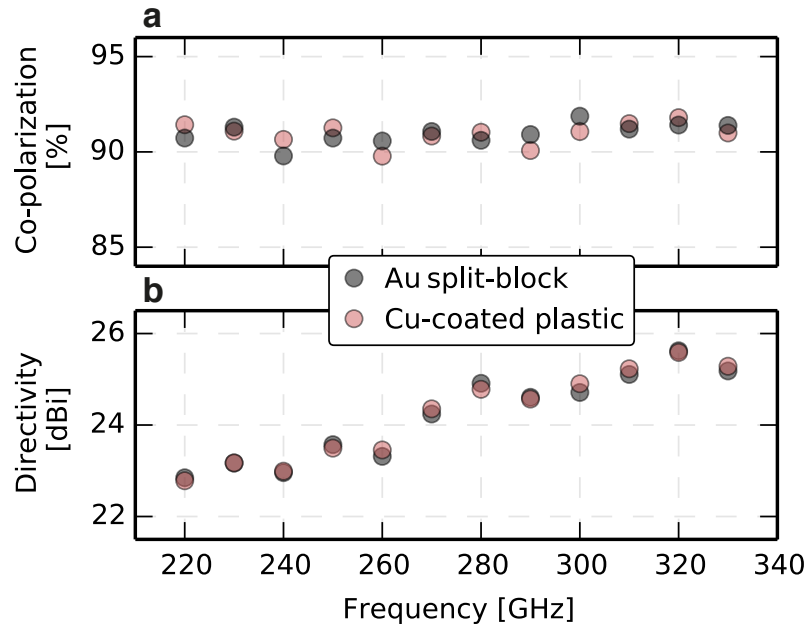

Fig. 3. (a) Co-polarization content of the total power emitted and (b) directivity as a function of frequency.

( $-30 \mathrm{~dB}$ vs. $-25 \mathrm{~dB}$ for the split-block horn). This agreement is further corroborated by the analysis of the co-polarization content as well as the directivity as shown in Fig. 3(a) and (b), respectively. Both horns exhibit a high co-polarization content close to the theoretical maximum of $90.5 \%$ and a directivity approaching $26 \mathrm{dBi}$ at the upper band edge.

These results show that the plastic horn provides the same performance as the conventionally machined horn, again underlining the quality of the fabrication technique used.

\section{SUMMARY}

In summary, we have developed and successfully demonstrated an SLA-based fabrication process suited for MMW components up to $330 \mathrm{GHz}$ and possibly beyond. Given the convincing performance of the characterized components, and the advantages in terms of flexibility, speed and weight, metalcoated plastic components represent a competitive alternative to conventionally machined components for both prototyping and production. Future investigations will focus on more complex geometries, such as monolithic orthomode transducers, which benefit even more from the versatility of the process.

\section{ACKNOWLEDGMENT}

The authors would like to thank M. Billod and M. Favre of SWISSto12 for help with the manufacturing and Dr. J. L. Hesler of Virginia Diodes Inc. for providing the reference diagonal horn antenna.

\section{REFERENCES}

[1] A. Macor, E. de Rijk, S. Alberti, T. Goodman, and J.-P. Ansermet, "Note: Three-dimensional stereolithography for millimeter wave and terahertz applications," Review of Scientific Instruments, vol. 83, no. 4, p. 046103, 2012.

[2] P. T. Timbie, J. Grade, D. van der Weide, B. Maffei, and G. Pisano, "Stereolithographed MM-wave corrugated horn antennas," in Infrared, Millimeter and Terahertz. Waves (IRMMW-THz), 2011 36th International Conference on, 2011, p. 13.

[3] J. F. Johansson and N. D. Whyborn, "The diagonal horn as a submillimeter wave antenna," Microwave Theory and Techniques, IEEE Transactions on, vol. 40, no. 5, p. 795800, 1992. 